\title{
Abstracts of the meeting of the Clinical Genetics Society held at the Royal College of Physicians, London on 5 and 6 December 1986
}

\section{Visit of Health Minister to Clinical Genetics Society}

Baroness Trumpington, Under-Secretary of State for Health, addressed the scientific meeting of the Clinical Genetics Society at the Royal College of Physicians on 5 December 1986. (Abstracts of the talks and posters follow.) The Minister listened to several talks by members and stayed on for lunch, discussing informally a variety of topics, including the development of genetic services, genetic education, and genetic nurses.

Her speech (reported in full below) emphasised precisely those aspects of medical genetics that most of us would see as most important. She noted in particular the relevance of counselling against a background of the social changes that have led patients to expect to share more closely in decisions that affect their health including, of course, parenthood. Further, ". . . molecular biology harnessed in the service of the "new genetics' . .." was of greatest value when combined with expert counselling when there was a great new potential for the relief of anxiety and morbidity associated with the fear of genetic disease. The correction of misinformation, which might include exclusion or reduction of risk, brings benefit to many more people than will actually develop genetic disease. Prenatal diagnosis “. . . has a necessary role, if reproductive choice is to be sufficiently and precisely informed" and the prevention of an increasing number of genetic disorders is possible. Even when this is not so "genetic knowledge may provide ways of alleviating the effects of genetic disease, by treatment or by preparing the ground for care and support ....". This included, in the Minister's view, the need to bring together much personal health data on families to "ensure effective continuing and anticipatory care . . yet at the same time safeguard[ing] confidentiality".

Particularly heartening were the Minister's references to the recent Joint Statement from the Royal Colleges (Physicians of London, Pathologists, Obstetricians and Gynaecologists, and General Practitioners) advocating the rational introduction of advances in molecular biology and the provision of properly balanced Regional Genetic Services.

Although decisions on resources allocation remain largely the responsibility of individual Health Authorities, Regions are now asked each year by the DHSS about their plans for genetic services and Lady Trumpington's speech is an important signal of central recognition of the value of developments in Health Service genetics. However there is fierce competition for funds and it is imperative that local strategies be developed by clinical and laboratory geneticists working in unison. The relevant Health Service Managers must be personally convinced of the value of genetics, encouraged by the knowledge that the Minister agrees.

RODNEY HARRIS

Department of Medical Genetics, St Mary's Hospital, Manchester M13 OJH.

\section{Address given by Lady Trumpington, Under-Secretary of} State for Health in the House of Lords

(1) I am delighted to have the opportunity of speaking at this meeting of the Society, to give very much the layman's view of your specialty and its work. I am also conscious of telling grandsons and grand-daughters present here today, how to suck eggs. It would be unwise for me to attempt to explore the bewildering achievements of molecular biology or the methods it has developed. But I do see that these can be applied to a better understanding of genetic disease; to diagnosis, to treatment, and most important of all, to the prevention of inherited disease. Neither should I be reticent in acknowledging the humane intention which motivates this work and the purposes to which it is directed.

(2) It seems to me very likely that if a disease runs in families then members of those families, unless they are widely scattered, will come to recognise the fact. What they cannot know quite so easily are the rules by which it is transmitted, and therefore who among them might develop the disease, or pass it on to their children. After all, it wasn't obvious to anyone before Mendel, who, with the help of his gardeners, started the whole enterprise, by establishing the laws that bear his name.

(3) How very perplexing it really must be, therefore, for members of an affected family, to understand what is happening among them, and even more difficult for them to see what might be done about it. The combination of bewilderment, misunderstanding, and misinformation is a source of deep and sustained anxiety, which may permeate the affected family. I have personally met families who, not having had the benefit of expert counselling, have had two or maybe three children, suffering from mental and physical handicaps, each in the hope that the child might not suffer the same genetic disease as its older brothers and sisters. Having witnessed the heartache this brings I need 
no convincing that one of the great contributions to be made by clinical genetics is reducing such distress through well informed counselling.

(4) As I understand the matter, progress in molecular biology harnessed in the service of the 'new genetics', allows discovery of those members of a family who are likely to carry a particular gene and those who are not. And this estimate can be made with a precision that sometimes approaches certainty.

(5) Already it is possible to follow the passage, through certain families, of the genes whose message causes some of the most wretched of diseases. Among them are cystic fibrosis, muscular dystrophy, Huntington's chorea, haemophilia, the blood diseases that affect many people of Mediterranean or African or Far Eastern ancestry, and also the fault shown recently to be a common cause of severe mental retardation.

(6) I believe we may predict with some confidence the identification of genes responsible for many more rare diseases. This will remind us that the concerns of affected families are the ways in which a disease affects them, not the rarity of the disease. Diseases associated with several or many genes, including heart disease, diabetes, and even some cancers, will then yield to scrutiny. In due course, but I suspect some way off, the congenital defects which have a genetic element, and affect so many newborn babies, and which are a major cause of perinatal death and morbidity, may also be understand.

(7) Let me recount some of the benefits that may follow the discovery of this kind of genetic knowledge. I have just mentioned the correction of misinformation, and for some individuals, we can now offer the exclusion or reduction of their risk. This, of course, is a form of prevention-the removal of a morbid cause, with benefit to more people, I believe, than would actually develop the disease. Secondary prevention, especially of the common diseases I have referred to, offers means for couples to ensure that a disease is not passed on to their children. Failing that, genetic knowledge may provide ways of alleviating the effects of the disease, by treatment or by preparing the ground for care and support, through medical and social agencies.

(8) I suppose that if clinical genetics were able to do no more than tell individual members of an affected family that they were not themselves at risk of suffering the disease, or of passing it on to their children, that contribution alone to medical care would have been remarkable. But the achievement is much more remarkable. Even those who suspect that they are carriers and know their own risk, may ensure that their children are not affected.

(9) I understand that because of the way in which genetic characteristics are passed on, exact information on the genetic make-up of an individual cannot be obtained before conception. For this inescapable reason, prenatal diagnosis has a necessary role, if reproductive choice is to be sufficiently and precisely informed. The prospect of preventing the birth of a disabled child is bound to be accompanied by difficult and painful choices. It is a task of clinical geneticists to offer counselling in these circumstances.

(10) Let me say a few words about the social and political context in which these opportunities for the prevention of inherited disease have emerged. There is an increasingl high expectation that children should not be mentally of? physically disabled, and that all possible steps should be taken to ensure that this is so. The new genetics has heightened awareness of choice to this end. At the samen time there has been a shift in the relationship betweerad patients and their doctors. Patients have come to expect to share more closely in decisions that affect their healtis including, of course, parenthood. The purposes of clinica 5 genetics are especially relevant in this setting.

(11) Clinical genetics has developed ways of giving precis $\overrightarrow{\vec{b}}$ information to, and counselling, individuals at risk. Coung selling, I understand, means describing the choices avail able, the means of implementing them, and the possiblo outcomes of those choices. This draws on clinical experi ence, the experience of other patients in a similar dilemmai and a sure knowledge of disease. Such activity is noin unique to clinical genetics; indeed there is an obvious senser in which counselling has always been central to the practice of medicine, but the circumstances of genetic counselling have a poignancy which surely bring out its most valuable qualities. Scrutiny of the qualities sought after, and mos? valued by patients, might yield benefits for the practice of medicine as a whole.

(12) Among the concerns of clinical geneticists, and one w⿳్乛𠃌 share, is the question of translating the developments of medical science into a comprehensive service for patientso The advances have been rapid; more rapid, I understand than even you yourselves have expected. Already the have begun to influence medical practice, increasing th range of clinical judgments made possible, and th expectations they arouse.

(13) Ministers are concerned to see that advances i molecular biology, which promise to have a major bearing on the way genetic advisory services are provided in future? are introduced and applied in the NHS on a rational basis.. Health services in their turn will want to make the necessary response.

(14) The basic elements of an effective genetics service arealready well understood. These have been described mos?. recently in a Joint Statement from the Royal College of Physicians, London, Pathologists, and Obstetricians an\& Gynaecologists to which the Royal College of Generat Practitioners have added their support. There must be clinicians of wide experience who are expert in genetics and inherited disease and those abnormalities which mat. have a genetic component. They must be skilled if counselling. They will also be acquainted with the new laboratory techniques and their application to diagnosis? The importance of training and education in a disciplin\& which is largely unfamiliar, but which will have aff increasing impact on almost all medical specialities an£ practice, is obvious. Much of the responsibility of th\&? training will naturally fall to clinical geneticists, particum larly those involved in undergraduate and postgraduate institutions.

(15) To be fully effective the organisation of genetio services must take into account the coordination that necessary between clinical genetics and other specialitie especially family practice, obstetrics, and paediatrics, an£ also with clinical and scientific laboratory specialities. 
(16) Genetic diseases are the concern of families, the members of which are often widely spread. Information on any one individual may be very important to the health of another individual. It means that there is a need to bring together much personal health data, in a way that will ensure effective continuing and anticipatory care for all members of a family, yet at the same time safeguard confidentiality.

(17) Although, for various reasons, there has been piecemeal evolution of genetic services, its direction has been maintained by the vigour and cooperation that are notable features of those who practice the speciality. I believe, too, that the professional cohesiveness of clinical geneticists has encouraged the development of an unusually clear and responsible view of the way forward. But already it is evident that the scope and pace of recent advances, and the impact they will make upon medical practice, require a larger strategy, a strategy which must be underpinned by wise medical advice.

(18) The orderly development of clinical services must also reflect the new opportunities which will flow predictably from medical advances. It is, of course, for health authorities to determine, in the light of the resources available to them, which services should be developed. They obviously need to use scarce resources efficiently, and avoid wasteful duplication.

(19) The thorough ground work, by you and your colleagues, on the practical needs for development of effective services, has provided a valuable resource for those managers and planners on whom the responsibility will fall. Members of your Society have also played an important part in bringing the applications of the new genetics to the service of patients: I should like to congratulate you and wish you very well in the future.

\begin{abstract}
Age at onset in multiple endocrine neoplasia, type 2a (MEN 2a) and implications for screening

B A J PONDER, D EASTON, R COFFEY, M A PONDER, M E PEMBREY, M TELENIUS-BERG, $H$ TELENIUS, AND THE CANCER RESEARCH CAMPAIGN MEDULLARY THYROID GROUP Institute of Cancer Research, Sutton, UK, and Hassleholm, Sweden.
\end{abstract}

We have calculated the probability that a gene carrier of MEN 2a will (a) have been diagnosed from clinical presentation or (b) be diagnosable by stimulated calcitonin screening by a given age. The analysis is based on 68 affected members of $22 \mathrm{MEN} 2 \mathrm{a}$ families in the CRC Group MEN 2 register and 151 of their apparently unaffected first degree relatives. An arbitrary age distribution was postulated and used to calculate the combined genetic and age related risk for each subject in the pedigrees. From these values a second distribution for age at presentation was calculated and substantiated for the first. The process was repeated until the distributions converged. The proportion of MEN 2a gene carriers who will have been diagnosed following clinical presentation is estimated to be $20 \%$ by age $25,65 \%$ by age $50(95 \%$ confidence limits 53 to $71 \%$ ), and $80 \%$ by age 70 . On stimulated calcitonin screening, $50 \%$ of gene carriers have converted to positive by age 12 and $90 \%$ by age 20 . The clinical penetrance of MEN 2a is incomplete to an extent which is significant for decisions about family screening. Having healthy elderly parents does not necessarily exclude familial disease in a patient with apparently sporadic MTC. The age at onset data may be used in combination with the family structure to estimate the risk for a given individual.

\section{Neurofibromatous neuropathy}

P K THOMAS, R H M KING, R CHIANG, A K SHARMA, AND A W DOWNIE

Royal Free Hospital School of Medicine, London, and University of Aberdeen, Scotland.

Neurofibromatosis may give rise to focal deficits of peripheral nerve function related to the development of localised lesions of cranial nerves, spinal roots, limb girdle plexuses, or peripheral nerve trunks. We have observed three cases with an adult onset of a slowly progressive, distal, symmetrical, sensorimotor neuropathy in three patients with neurofibromatosis, one of whom had bilateral acoustic neurinomas. Nerve conduction studies showed depression or loss of sensory nerve action potentials and a moderate reduction in motor nerve conduction velocity. In all three cases there was a diffuse nodular enlargement of the peripheral nerves and in one myelography showed that the enlargement also involved the spinal roots. Nerve biopsy showed neurofibromatous changes in all three cases. The term neurofibromatous neuropathy is suggested for this syndrome, which may represent a separate disease entity. It differs from cases of neurofibromatosis associated with peroneal muscular atrophy that have been described (W G Bradley et al, Brain 1974;97:521; E P Bosch et al, Neurology (Minneap) 1981;31:1408) but it resembles the patient described by Ohnishi and Nada (Acta Neuropathol (Berl) 1972;20:258).

\section{Tuberous sclerosis: a linkage study}

A E FRYER*, A CHALMERS*, J NOADES $\dagger$, J P OSBORNE*, $S$ POVEY $\dagger$, D SEEDBURGH $\dagger$, AND J R W YATES $\ddagger$

*The Royal United Hospital, Bath; +MRC Human Biochemical Genetics Unit, University College, London; and $¥$ the Duncan Guthrie Institute of Medical Genetics, Yorkhill, Glasgow.

A linkage study in tuberous sclerosis using blood groups, plasma proteins, and red cell enzyme polymorphisms is in progress. Families have been obtained through the Tuberous Sclerosis Association of Great Britain, clinical geneticists, paediatricians, neurologists, and dermatologists in England and Wales. Currently, seven three generation families and 11 two generation families have been fully documented. All affected members fulfil Gomez's diagnostic criteria and the disease is excluded from non-affected members by physical examination, Wood's light examination, direct and indirect fundoscopy, cranial CT scan, renal ultrasound, and skeletal survey. Subjects are excluded 
from the linkage calculations if doubt exists about the diagnosis. Lod scores to date are positive with the ABO blood group $(\hat{z}=2 \cdot 3, \hat{\theta}=0)$ and adenylate kinase $(\hat{z}=1 \cdot 2$, $\hat{\theta}=0$ ) which form a linkage group on distal $9 q$.

\section{Sex and cause of neural tube defects \\ MARY J SELLER \\ Paediatric Research Unit, Guy's Hospital, London.}

Overall, NTD occur more commonly in females than males. However, I will show that if NTD are separated according to the site of the lesion along the neural tube, the sex ratio varies; in one site it approaches unity, in others the female excess is very marked, while in the case of low spinal lesions there is a pronounced male bias. Sex ratios in NTD are therefore not a random distribution, and this must have implications in the pathogenesis of the lesions. In embryogenesis, the neural tube is formed by two processes, neurulation, which produces the brain and spinal cord down to the upper lumbar region, and canalisation, which creates the most caudal parts. It seems that males and females have differing abilities to perform successfully these two processes. With regard to neurulation, I will show that in the mouse, at the time of neurulation, there are observable differences between male and female embryos; females are less advanced developmentally than males. I propose therefore that growth retardation is involved in the genesis of neurulation type NTD.

DNA analysis in risk estimation for common genetic disorders

S V HODGSON, A WALKER, K A HART, C G COLE, L JOHNSON, $\checkmark$ DUBOWITZ*, AND M BOBROW

Paediatric Research Unit, Prince Philip Research Laboratories, Guy's Tower, London SE1 9RT; and *Department of Paediatrics and Neonatal Medicine, Hammersmith Hospital, London W12 OHS.

Two hundred and seventy-eight families registered with a diagnosis of Duchenne (DMD) or Becker (BMD) muscular dystrophy were approached and offered counselling on the implications of linkage analysis using DNA polymorphisms for carrier detection and prenatal diagnosis. About $30 \%$ of families responded to the initial invitation, but during the subsequent two years all but six of these families requested counselling. The acceptability of genetic counselling and linkage diagnosis does not appear to be a major obstacle. An average of approximately five persons have been tested per family and two people per family still await testing. The number of DNA digests per family is just under nine; for those cases presenting when already pregnant, the comparable figure is 22 . Estimated immediate costs work out at $£ 1000$ to $£ 2000$ per family. We have probably tested over $10 \%$ of the current total UK Duchenne families. Handling the cases so far not investigated would perhaps cost $£ 2$ to 3 million. This may represent 5 to $10 \%$ of all Mendelian diseases amenable to single linkage analysis. Twenty-five pregnancies at high risk for DMD have been referred for testing, as a result of which seven have been terminated.

Audit of medical genetic contribution to clinical manage ment in the neonatal period

CAROLE MCKEOWN, JOANNA DAWSON AND DIAN DONNAI St Mary's Hospital, Manchester M13 OJH.

In order to assess the contribution of clinical geneticists to neonatal management, a review of 125 liveborn babie referred for diagnostic opinion over a two year period waร performed. Seventy-four babies $(59 \%)$ were seen at $\mathbf{S} \bar{b}$ Mary's, 43 inborn (19 originally booked elsewhere) and $3 \mathrm{~N}$ outborn and transferred for neonatal intensive care or surgery. Fifty-one $(41 \%)$ were seen at 10 other hospitals ip the region. All babies were seen within 24 hours ofs referral. In $104(83 \%)$ an exact diagnosis was made eitheb immediately or after appropriate investigations; $15(12 \%$ had an unrecognised pattern of anomalies and six $(5 \%$ were considered normal. In only $54(43 \%)$ had the actuas diagnosis been considered in the differential diagnosis af. referral. The increased numbers of diagnoses after the genetic consultation affected management in several ways: deferred surgery, earlier surgery, the search for possible associated anomalies, and the saving of cell lines fof further investigations. Seventy-two $(58 \%)$ families wers subsequently referred for formal genetic counselling and $\overline{\bar{O}}$ as a third of babies had died, the early diagnostic referrad was also an invaluable opportunity to obtain information relevant for counselling.

The impact of DMD DNA probes on reproductive decision L KERZIN-STORRAR, D DONNAI, M SUPER, C MCKEOWN, A P READ, AND R HARRIS

Department of Medical Genetics, St Mary's Hospital, ant Royal Manchester Children's Hospital, Manchester.

A regional genetic register of Duchenne muscular dysể trophy (DMD) families was established in Manchester i 1979. Studies with linked DNA polymorphisms began in 1983 and to date 105 of 147 families on the register hav? been studied. Significant alteration of carrier risk and th potential for prenatal exclusion or diagnosis has been madepossible in a large number of families. We present here oû experience with a first cohort of 33 women of reproductive age who have been recounselled after DNA studies, an report alterations in their reproductive decisions. We havE found the clinical impact of DNA linked polymorphisms iळ DMD families to be profound, both to those at low ris who now feel reassured to carry on with pregnancy without prenatal tests, as well as to those at high risk who now find their choice of acceptable options increased. We have observed that the range of 'acceptable risk' is influenced bt

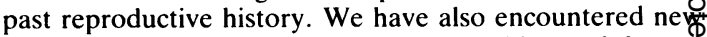
counselling dilemmas, particularly the sudden and dramą tic alteration of risks based on probe results in wome 8 whose previous reproductive actions were based on verg different risks. 
The application of closely linked restriction fragment length polymorphisms in counselling families with myotonic dystrophy

P W LUNT, A L MEREDITH, S M HUSON, M SARFARAZI, AND P S HARPER

Section of Medical Genetics, University of Wales College of Medicine, Cardiff.

DNA probes coding for the Apo C2 gene and closely linked to the locus for myotonic dystrophy (DM) can now be used to counsel families with this disorder. The success of this application has been assessed by a study of 46 families with DM referred to us for genetic counselling by means of linkage analysis. The prerequisites for counselling using closely linked probes are a suitable family structure and also full clinical testing of apparently asymptomatic members. In those families where we have used at least four different RFLP systems, 10/15 (67\%) of currently asymptomatic but at risk subjects referred and $17 / 22(77 \%)$ of other at risk subjects from these same families could be given a significantly altered estimation of their age dependent risk of carrying the DM gene. Allowing for up to $50 \%$ of untyped spouses proving heterozygous, prenatal detection could be offered to $9 / 21$ $(43 \%)$ couples referred and to $9 \cdot 5 / 24(40 \%)$ of other subjects from the same families. Results for the different RFLP systems can be combined for haplotype counselling, but there is an excess of homozygosity due to linkage disequilibrium between the various polymorphic alleles, with two haplotypes accounting for $83 \%$ of those observed.

Early experience using DNA probes in cystic fibrosis M SUPER, R ELLES, M SCHWARZ, A P READ, AND R HARRIS Department of Medical Genetics, St Mary's Hospital; and Clinical Genetics Unit and CF Clinic, Royal Manchester Children's Hospital, Manchester.

Results of testing 49 families showed that 18 were informative with Met $\mathrm{H}$ and $\mathrm{D}$ alone and 14 more with a combination of Met $\mathrm{H}$ and D with pJ3.11. Twelve of the remainder are informative: for $\mathrm{pJ} 3 \cdot 11$ alone (2), Met $\mathrm{D}$ on Banl digests (2), or for the more distantly located polymorphisms alone or in a combination. Five families were partly informative with a combination of all these probes: none was completely uninformative. The haplotype Met $\mathrm{H} 2$, Met $\mathrm{D}, \mathrm{pJ} 3 \cdot 11$ occurred much more often in the non-CF chromosome of each parent than in $C F$ chromosomes. There appears to be linkage disequilibrium between $C F$ and the $7 \cdot 5 \mathrm{~kb}$ allele of Met $\mathrm{H}\left(\chi^{2}=4 \cdot 6\right.$, $\mathrm{p}<0 \cdot 05, \mathrm{n}=224$ chromosomes). Couples who had decided to have no further children are planning pregnancies, encouraged by the new tests.

\section{Confirmation of diagnosis of cystic fibrosis in second trimester abortuses by use of RFLP analysis}

ANN CURTIS AND DAVID BROCK

Human Genetics Unit, University of Edinburgh,
Edinburgh.

Microvillar enzyme assay in second trimester amniotic fluid has been extensively used in prenatal diagnosis of cystic fibrosis (CF) in pregnancies with a 1 in 4 risk of recurrence. Mothers with abnormal enzyme values usually opt for termination of pregnancy. Methods for confirmation of diagnosis by measurement of meconium albumin and protease levels in the abortus have a degree of uncertainty. The proportion of fetuses predicted to have $\mathrm{CF}$ is greater than $25 \%$, suggesting that there may be unrecognised false positives. If there is a living affected child in the family, it should be possible in principle to confirm diagnosis by RFLP analysis. We show that adequate samples of undegraded DNA can be extracted from fetal gut and other tissues, stored at $-20^{\circ} \mathrm{C}$ for periods of several years. In the first six cases analysed, diagnosis was confirmed in four abortuses and not confirmed in one. In the other case the markers were not fully informative. The use of RFLP analysis in an abortus to establish the phase relationships of the markers for subsequent first trimester prenatal diagnosis is technically possible, but should be approached with some caution.

Exclusion tests in pregnancy for Huntington's chorea: clinical use of the DNA probe G8

O W J QUARRELL, A L MEREDITH, A TYLER, S YOUNGMAN, L LAZAROU, AND P S HARPER

Section of Medical Genetics, University of Wales College of Medicine, Cardiff.

As a precursor to full predictive testing, couples with one partner at $50 \%$ risk for Huntington's chorea have been considered for exclusion tests in pregnancy. This can allow couples to have children at low risk (maximum 5\%) without altering their own risks. Forty-six couples have made enquiries regarding the use of DNA studies and Huntington's chorea. Two couples would not contemplate a termination in any circumstances and for one couple there were difficulties in obtaining a blood sample from the affected parent. The remaining 43 couples all decided to give blood for DNA typing. After this counselling session couples could withdraw from the study or attend for a second counselling session or might become pregnant before knowing the results on informativeness. Twentytwo couples have been followed up so far, of whom 12 still considered an exclusion test to be their best option. Using five restriction enzymes with the G8 probe approximately $2 / 3$ couples are currently informative, but this proportion will increase as further polymorphisms and new probes become available. There have been six pregnancies in this series so far. Two couples were already pregnant at presentation but, despite this, there was time to arrange at least two counselling sessions and DNA studies. Three couples were uninformative, one couple withdrew from the study when pregnant, and two couples have had chorionic villus sampling.

Localisation of the gene for steroid sulphatase deficiency (X linked ichthyosis)

D R GOUDIE, E F GILlARD, J R W YATES, D AITKEN,

N A AFFARA, P TIPPETT, AND M A FERGUSON-SMITH

Duncan Guthrie Institute of Medical Genetics, Yorkhill, vilasgow G3 $8 S J$

Seven families with steroid sulphatase deficiency (STS) 
were studied with the polymorphic DNA markers 782 (DXS85), dic56 (DXS143), GMGX9, and with Xg blood grouping. The linkage relationship of these markers was also studied in six normal three generation families. The carrier status of females in the steroid sulphatase deficient families was determined by assay of steroid sulphatase activity in hair roots. The GMGX9 marker, which was isolated from a flow sorted $\mathrm{X}$ chromosome library, was deleted in all affected subjects in six of the seven STS deficient families. Two point linkage analysis gave the following results.

$\begin{array}{lcc} & \hat{Z} & \hat{\theta} \\ \text { STS v GMGX9 } & 8 \cdot 7 & 0.00 \\ \text { STS v 782 } & 5 \cdot 8 & 0.08 \\ \text { GMGX9 v dic56 } & 4.9 & 0.07\end{array}$

In a male with STS deficiency due to a deletion of distal $\mathrm{Xp}$, both dic56 and 782 were retained while GMGX9 and $\mathrm{Xg}$ were deleted. This provides evidence for the order pter-Xg-(STS, GMGX9)-dic56-782-D ${ }_{2}$-cen. This order was consistent with the minimum number of recombinaions in families informative at three or more marker loci.

Population genetics implications of the premutation hypothesis for the generation of the fragile $X$ mental retardation gene

R M WINTER

Division of Inherited Metabolic Disease, MRC Clinical Research Centre, and Kennedy-Galton Centre, Harperbury Hospital, Radlett, Herts.

The population genetics implications of the premutation hypothesis for the generation of the fragile $\mathrm{X}$ mental retardation gene are explored. With some broad assumptions, the consequences of the model are that (a) $50 \%$ of mothers of probands carry the premutation, (b) $6.5 \%$ of mothers of probands receive the premutation from their mothers, $18.9 \%$ from their fathers, and $24.6 \%$ as a 'new mutation'. (c) The incidence of carriers for the full mutation equals the incidence of affected males, whereas the incidence of carriers for the premutation is 1.35 the incidence of affected males. (d) Assuming mutation rates are equal in eggs and sperm, the average mutation rate from normal to premutation alleles is $1.67 \times 10^{-4}$. (e) The expected segregation ratio in sibs of probands is 0.44 which corresponds to observed values. In addition, predictions using the premutation hypothesis of the expected segregation ratio in sibs of mothers of probands fits well with the data of Vogel et al (Hum Genet 1985;71:1-6).

Hypomelanosis of Ito and diploid/triploidy DIAN DONNAI, A P READ, CAROLE MCKEOWN, AND - TONY ANDREWS

Department of Medical Genetics, St Mary's Hospital, Manchester M13 OJH.

Three unrelated patients with hypomelanosis of Ito (HI) were studied. Two had skeletal anomalies similar to those seen in triploid fetuses. Skin biopsy was performed an histological and cytogenetic studies carried out on culturey fibroblasts. All cases had normal cytogenetic findings lymphocytes, but the two children with skeletal anomalies had mixed diploid and triploid cells in skin fibroblasts. Published clinical descriptions of $\mathrm{HI}$ and diploid/triploig cases show considerable overlap. Our findings suggest botd that HI comprises a heterogeneous group of mosaics anfa that clonal mosaics other than those resulting fro $\vec{B}$ differential lyonisation may cause skin abnormalities following Blaschko's lines. Autosomal dominant inheritances of $\mathrm{HI}$ has been suggested, but review of published repors? and our findings do not support this. The genetic counsel ing implications are that the risk of recurrence if the proband is male is nil; if the proband is female the risk if probably also low but an X linked mutation should be considered.

Studies on the origin of hypomelanosis of Ito A P READ, DIAN DONNAI, CAROLE MCKEOWN, AND TONY ANDREWS

Department of Medical Genetics, St Mary's Hospitad Manchester M13 OJH.

DNA fingerprints were obtained from blood and skin $\mathscr{O}$ three patients with hypomelanosis of Ito and from the blood of their parents. In all three cases, the band patterris obtained from blood and skin DNA were identical and each parent had bands which were not present in the child Cases I and II have pure diploid blood but mixe diploid-triploid skin. Our data show this is not due 18 chimerisms or failure of the first meiotic division. maternal 1qh + chromosome is present in a single copy i both diploid and triploid cells of case 1, ruling out second division failure in the egg. In case III all cells were $46, X$ Blood groups, HLA types, and X chromosome DNC polymorphisms showed no evidence of chimerism. Thi boy is probably mosaic for a post-zygotic point mutation.

Clinical, cytogenetic, biochemical, and molecular investig tion of a case of mosaic tetrasomy 12p

D A AITKEN, J GALT, P BATSTONE, T REBBECK, E BOYD,

M E FERGUSON-SMITH, AND $J$ R W YATES

Duncan Guthrie Institute of Medical Genetics, Yorkhill, Glasgow G3 8SJ.

Chromosome analysis was performed on a dysmorphic newborn female with hypotonia and seizures. Birth weigth and head circumference were on the 50th centile but leng was on the 10th. The baby had a striking posteris distribution of scalp hair, large anterior fontanelle, prominent forehead, hypoplastic supraorbital ridges, flat facieș upward slanting palpebral fissures, hypertelorism, sho: nose with anteverted nares, thin upper lip, flabby ef lobes, excess nuchal skin, short neck, coccygeal tail with sacral pit, some shortening of the limbs, short hands with stubby fingers, and small feet. Lymphocyte cultures 
showed a 46,XX karyotype. Cultured fibroblasts derived from a skin biopsy taken at four weeks of age revealed the presence of mosaicism with an additional small metacentric chromosome in all metaphases examined. The child died at eight months and the chromosome abnormality was confirmed post mortem in samples of lung, liver, peritoneum, and pericardium. The additional chromosome was tentatively identified as an isochromosome for the long arm of chromosome 21 , but subsequently no evidence of enhanced hybridisation was found with a chromosome 21 specific DNA probe. Further studies with the Kirtsen-ras probe (mapped to $12 p$ ) suggested that the additional chromosome material may be derived from the short arm of chromosome 12. This was confirmed by the demonstration of tetrasomic gene dosage for two genes mapped to $12 \mathrm{p}, \mathrm{LDH}-\mathrm{B}$ and GAPDH.

A follow up study of 80 handicapped school leavers interviewed five to seven years after genetic counselling MARY VOWLES

Exeter and South-West England.

Home visits were made to 22 deaf, 29 visually handicapped, and 29 physically handicapped students. Pedigrees were updated including their own marital status and offspring. A total of $77 \%$ recalled the initial interview and $65 \%$ the risks. Whether they were distressed or not and considered that interview helpful or not, both at the time and since, was discussed. Their parents' and sibs' attitudes to counselling and their own present attitude in view of further discussion at follow up were totally favourable with one exception. Further genetic advice from a doctor other than their GP had been sought or received by six out of the 80 studied.

Localisation of the gene for congenital adrenal hypoplasia J R W YATES, E F GILLARD, A COOKE, J M COLGAN, $T$ J EVANS*, AND M A FERGUSON-SMITH

Duncan Guthrie Institute of Medical Genetics, Yorkhill, Glasgow; and *Royal Hospital for Sick Children, Glasgow.

Two families with $\mathrm{X}$ linked recessive congenital adrenal hypoplasia (AHC) have been studied. In family 1 the affected boy was mentally retarded and chromosome analysis showed a deletion of Xp21 in the boy and his mother, which was confirmed by flow cytometry. The probes C7 (DXS28) and pB24 (DXS67) were deleted but not pERT87-30 (DXS164) or p99-6 (DXS41). This patient did not have glyceroluria or raised creatine kinase. In family 2 four males from a sibship of nine males and three females were affected by AHC not associated with intellectual impairment. No deletion was identified but pB24 segregated with the disease. Our findings support localisation of $A H C$ to $\mathrm{Xp} 21$. There are patients reported with AHC, glycerol kinase deficiency (GK), and Duchenne muscular dystrophy (DMD) due to deletions which are proximal to $\mathrm{pL} 1(D X S 68)$ and which do not involve $\mathrm{C} 7$ or pB24. Taken together with our first case this maps $A H C$ between pERT87-30 and pL1 and supports the order cen-DMD-GK-AHC-pter.

Analysis of perinatal mortality by ethnic group: does consanguinity contribute to mortality due to congenital malformations?

L $S$ DUNLOP AND R M WINTER

Kennedy-Galton Centre, Harperbury Hospital, Harper Lane, Radlett, Herts.

Congenital malformations account for a significant proportion of perinatal deaths. This study was undertaken to assess their contribution in different ethnic groups and to attempt to determine the extent to which autosomal recessive conditions are an important factor. The maternity and neonatal unit records of four hospitals in the North West Thames Region, for a six year period from the beginning of 1980 to the end of 1985 , were reviewed in order to ascertain all stillbirths and neonatal deaths. The deaths were classified according to the Wigglesworth classification by consulting necropsy reports, clinical radiographs, and photographs where possible. The four hospitals studied notified the precise ethnic group of the mothers. Hence it was possible to analyse the data by ethnic group. The population studied had a total number of births of 63442 with 803 perinatal deaths, giving an overall perinatal mortality rate of $12 \cdot 6 / 1000$. When broken down into ethnic groups the rates were: Caucasians $11 \cdot 2 / 1000$, Indian Asians $12 \cdot 4 / 1000$, and Pakistani $15 \cdot 4 /$ 1000. The Pakistanis had a higher rate of lethal congenital malformations $(6 \cdot 3 / 1000)$ and recessive conditions $(1 \cdot 71 /$ 1000), compared with Indian Asians (3.3/1000) and (0.29/ $1000)$ and Caucasians $(2 \cdot 9 / 1000)$ and $(0 \cdot 12 / 1000)$. Recessive conditions occurred more commonly among consanguineous couples. These results show similar trends to those reported by other authors and emphasise the fact that Asians, particularly Pakistanis, are at greater risk of congenital malformations and perinatal death and should be treated as a high risk group.

Genetic linkage studies in Charcot-Marie-Tooth disease: failure to demonstrate linkage relationships to chromosome 1 markers

DHAVENDRA KUMAR

Centre for Human Genetics, Sheffield.

A genetic linkage study was performed in five families with autosomal dominant Charcot-Marie-Tooth disease (CMTD) of slow motor nerve conduction velocity type (HMSN type 1). Previous studies in other families have shown positive linkage of the HMSN type 1 gene to the Duffy (Fy) blood group locus on the long arm of chromosome 1 (1q23). The present results (three families) do not favour close linkage to the Fy locus $(Z=-0.485$, $\theta=0 \cdot 0)$. Linkage to other chromosome 1 markers, $R h$ $(\mathrm{Z}=-2 \cdot 150)$ and PGM1 $(\mathrm{Z}=-1 \cdot 520)$, is also excluded $(\theta=0 \cdot 05)$. Linkage studies in two informative families with DNA probes (AT3 and Apo A2) encoding restriction 
fragment length polymorphisms on chromosome 1 were not informative for linkage. Two families were informative for linkage to the $\mathrm{P}$ blood group and the glyoxalase 1 (GLO1) loci on chromosome 6 . Linkage was neither confirmed nor rejected $(Z=0.588$ and $0.416, \theta=0.0)$. The assignment of the HMSN type 1 gene to 1q23 may be considered provisional due to lack of firm evidence in favour of close linkage to the Fy locus. Demonstration of positive linkage in some families and lack of evidence for Fy:HMSN linkage in other families would support the concept of genetic heterogeneity in HMSN type 1. Further linkage studies using chromosome 6 markers are indicated.

\section{Linkage in a large family segregating for cleft palate}

O JENSSON, A ARNASON, A BJORNSSON, M FARRALL, A IVENS, J CHAMBERS, R WILLIAMSON, AND G MOORE

Genetics Division, The Blood Bank, Reykjavik, Iceland; and Department of Biochemistry, St Mary's Hospital Medical School, University of London, London.

A large Icelandic family has been studied in which cleft palate plus ankyloglossia segregates as an $\mathrm{X}$ linked Mendelian disorder. Males usually exhibit cleft secondary palate with ankyloglossia, while carrier females only show ankyloglossia, DNA samples were prepared from blood from family members and the segregation of approximately $30 \mathrm{X}$ chromosome probes was studied. Significant linkage was obtained with several probes localised to the proximal region of the long arm. It will be possible to isolate mRNA from palatal shelf tissue from terminations of pregnancy, and to study the expression of genes from this region of the $\mathrm{X}$ chromosome during the relevant stages of embryogenesis. This should provide a model for the study of mutations which can cause congenital defects and dysmorphologies.

\section{Posters}

Folate sensitive fragile sites on the $X$ chromosome EDNA L MALTBY

Centre for Human Genetics, Sheffield S10 5DN.

In the course of routine investigation in this laboratory for the fragile site at $\mathrm{Xq} 27 \cdot 3$ linked with heritable mental retardation, it has become apparent that there are several other folate sensitive sites on the $\mathrm{X}$ chromosome. These occur at a low frequency. Three such sites were demonstrated: $\mathrm{Xp} 22, \mathrm{Xq} 22 \rightarrow 23$, and once Xq27.1. The latter site is very close to the clinically important region and could be the reason why fragile Xqter can be reported as positive in unexpected circumstances.

Further localisation of the gene for $X$ linked hypophosphataemia

A P READ, $R$ V THAKKER, K E DAVIES, R C MOUNTFORD, A KING, M DAVIES, AND J L H O'RIORDAN

Department of Medical Genetics, St Mary's Hospital, Manchester; Department of Medicine, Middlesex Hospital, London; and Nuffield Department of Clinical Medicine, Oxford.

Previously reported and new families with $\mathrm{X}$ linked hypophosphataemic rickets (HPDR) were studied using $\stackrel{\overparen{\Phi}}{:}$ probes 782, D2, 99-6, C7, pERT87, and 754, which define $\vec{\equiv}$ loci $D X S 85, D X S 43, D X S 41, D X S 28, D X S 164$, and $\stackrel{9}{\hookrightarrow}$ $D X S 84$ respectively. For $D X S 41$ and $D X S 43$ the maximum 0 two point lod scores with $H P D R$ are 7.34 at $10 \mathrm{cM}$ and 4.14 व at $15 \mathrm{cM}$ respectively. Several families show three point $\overline{\bar{\sigma}}$ crosses suggesting the order $D X S 43-H P D R-D X S 41$. Five $\mathbb{Q}$ locus mapping with the LINKMAP programme gave relative likelihoods of $2 \cdot 6: 8 \times 10^{5}: 1$ that the HPDR gene is in the intervals $D X S 85-D X S 43, D X S 43-D X S 41$, and $\vec{\circ}$ $D X S 41-D X S 84$ respectively. Bridging markers can now be defined with confidence for gene tracking in families.

Identification of a second DNA polymorphism in the human low density lipoprotein receptor gene

ROHAN TAYLOR, BERNHARD HORSTHEMKE*, ALISON DUNNING, AND STEVE HUMPHRIES Charing Cross Sunley Research Centre, London W6 8LW; 응 and ${ }^{*}$ Institut für Humangenetik, Universitätsklinikum Essen, Hufelandstrasse 55, D-4300 Essen 1, Federal $\rightarrow$ Republic of Germany.

Familial hypercholesterolaemia is an autosomal dominant $\overrightarrow{0}$ disease affecting approximately 1 in 500 people and is $\stackrel{\infty}{v}$ caused by mutations in the LDL receptor gene. It is characterised by high levels of serum LDL cholesterol and therefore an increased risk of myocardial infarction and atherosclerosis before the age of 50 years. Genomic clones $\bar{O}$ for the $3^{\prime}$ region of the human low density lipoprotein (LDL) receptor were isolated from a phage library. The $\mathbb{Q}$ inserts from these clones were used as probes to screen DNA samples from a number of unrelated subjects for $\underline{ }$ restriction fragment length polymorphisms (RFLPs). Repetitive sequences in these probes were removed by preincubation with an excess of total human DNA. Using? this method we have identified an RFLP with the enzyme PstI, with an allele frequency of approximately $0 \cdot 5$. The variant site maps within approximately $5 \mathrm{~kb}$ from the $3^{\prime}$ 을 end of the translated region of the LDL receptor gene. This RFLP is in apparent linkage equilibrium with the previously reported PvuII RFLP. The use of the PvuII and $P$ stI RFLPs in conjunction as a haplotype will increase the availability of presymptomatic diagnosis of familial 윽 hypercholesterolaemia to approximately $68 \%$ of families.

The application of image-analysis techniques to the study of sperm chromosomes

P T TOMKINS, C V CARROLL, AND J A HOUGHTON Department of Microbiology, University College, Galway Ireland.

In recent years, we have instituted many changes in the original sperm penetration and chromosome visualisation우 assays, so that, with certain provisos, it is now possible to obtain sperm chromosome metaphases reliably. The de- $\mathbb{\mathbb { \Phi }}$ velopment of defined fertilisation and culture conditions using chemically uniform media has eliminated much of 
the inherent assay variation and ensured that, if optimal penetration is attained, then a high level of developmental progression through $\mathrm{G} 2$ to chromosome condensation will occur. However, successful analysis of final hybrid spreads is dependent on chromosome quality while the detection of small structural chromosome rearrangements depends on interpretable banding. The use of prefixation banding techniques that rely upon the incorporation of RNA polymerase inhibitors into post-S phase cultures readily produces $G$ band like patterns, but interpretation is made more difficult by the stretched diffuse appearance. Sperm chromosome images have been retained and manipulated using a microscope mounted TV camera, $512 \times 512 \times 8$ bit frame store coupled to an RM Nimbus computer. The use of edge enhancement, threshold expansion, and background blanking routines dramatically improves clarity and interpretation which is not lost after $35 \mathrm{~mm}$ screen photography. These techniques also make discrimination of the centromere stretching of 1,9 , and 16 encountered in human sperm chromosomes, as well as radiation induced breaks in the sperm chromosomes of radiotherapy patients, much easier. Image analysis aided studies of human sperm chromosomes are now in progress.

\section{A study of the prevalence of Huntington's chorea in the Grampian Region \\ SHEILA SIMPSON AND ALAN W JOHNSTON \\ Aberdeen Teaching Hospitals, Aberdeen.}

A survey of Huntington's chorea was undertaken in 1985 to 1986 in order to up-date the Huntington's chorea component of the Genetic Register. The number of previously identified families was 56 and is now 87 . A prevalence date of 30 January 1984 was chosen when 48 living persons were known. Using the census population (1981) of 462891 , a prevalence of $10 \cdot 37 / 100000$ was found.

Prevalence of hypertrophic cardiomyopathy in the Northern Region

C P BENNETT*, J BURN*, AND G MOORE $\dagger$

${ }^{*}$ Department of Human Genetics, University of Newcastle, Newcastle upon Tyne NE2 4AA; and +Department of Biochemistry, St Mary's Hospital Medical School, London.

Hypertrophic cardiomyopathy (HCM) is an often unrecognised cause of sudden death in children and adults. In many cases diagnosis relies on echocardiography. The condition is reported to be familial in $56 \%$ of cases. Family studies suggest an autosomal dominant mode of inheritance with variable expression and reduced penetrance. In the Northern Region (population 3.2 million), we have undertaken a prevalence study. Using multiple sources of ascertainment, the most profitable of which was the echocardiographic records, 338 suspected cases were identified and 143 of these had convincing evidence of HCM, 97 of whom were not known to have died. This puts the minimum prevalence at $3 \cdot 33 / 100000$. This is likely to be an underestimate but still represents an important clinical burden. The present phase of our study is to identify pedigrees suitable for linkage analysis so as to localise the gene or genes involved. Four potentially informative families have given blood. One of these has been probed using the calcitonin gene TaqI polymorphism in view of a report of $\mathrm{HCM}$ in a child with an $11 \mathrm{p}$ deletion and the evidence of calcium metabolism involvement in HCM.

Restriction enzyme analysis of mitochondrial DNA in patients with mitochondrial myopathy

A E HARDING, I J HOLT, AND J A MORGAN-HUGHES

Department of Clinical Neurology, Institute of Neurology, London.

It has been suggested that mitochondrial myopathy may be inherited by means of transmission of mutant mitochondrial (mt) DNA. In order to test this hypothesis we have analysed restriction fragment length polymorphisms in $\mathrm{mt}$ DNA from 63 subjects in 22 maternal lines containing 27 patients with mitochondrial myopathy. DNA was extracted from leucocytes and digested with 28 endonucleases recognising four, five, or six base restriction sites. The DNA fragments were electrophoresed, transferred to nylon membranes, and then hybridised with ${ }^{32} \mathrm{P}$ labelled HeLa cell mt DNA. Twelve of the 22 maternal lines containing patients with mitochondrial myopathy showed mt DNA polymorphisms at one or more sites, some of which occur at low frequency in the normal population. No differences were demonstrated in restriction site patterns between affected and unaffected subjects in the same maternal line, and no major deletions of mt DNA were observed in patients. This study thus provides no positive evidence of mitochondrial inheritance in mitochondrial myopathy but this has not been excluded.

DNA deletion screening in Duchenne and Becker muscular dystrophy

K A HART, A WALKER, C G COLE, S $v$ HODGSON, L JOHNSON, $\checkmark$ DUBOWITZ*, AND M BOBROW

Paediatric Research Unit, Guy's Hospital, London SE1 9RT; and *Department of Paediatrics and Neonatal Medicine, Hammersmith Hospital, London W12 OHS.

The DNA of more than 220 boys suffering from Duchenne (DMD) or Becker (BMD) muscular dystrophy was screened for deletions by probing with cloned DNA sequences from Xp21: pERT87-1, pERT87-8, pERT $87-15$, and pXJ1.1. These unique sequences are known to reveal DNA deletions in about $7 \%$ of DMD patients in a worldwide survey. We now have $18 \mathrm{DMD}$ deletion patients giving a similar deletion frequency. More than 35 BMD patients were screened in this survey. This disease is similar to, but less severe than, DMD. Both diseases have similar linkage relationships to $\mathrm{Xp} 21$ probes, yet no previous DNA deletions had been reported. Two BMD cases showed clear deletions and a third gave aberrant 
band sizes with pERT87-1. Further analysis has shown this to be a very small deletion of $\cong 6 \mathrm{~kb}$. Mild and severe Becker cases are represented by these deletions. DNA sequences commonly deleted in DMD patients are also deleted in BMD patients of varying severity, strengthening the evidence that they are allelic. In the long term, this observation may help in the understanding of the molecular pathology of these diseases. In the short term, further justification is provided for the application of DMD genetic counselling probes to BMD families.

The value of determining the male and female mutation rates in Duchenne and Becker muscular dystrophy using RFLPs

A WALKER, K A HART, C G COLE, S V HODGSON, L JOHNSON, V DUBOWITZ*, AND M BOBROW

Paediatric Research Unit, Guy's Hospital, London SE1 9RT; and *Department of Paediatrics and Neonatal Medicine, Hammersmith Hospital, London W12 OHS.

In order to ascertain the ratio of female to male mutation rates in the $\mathrm{X}$ linked recessive disorders Duchenne (DMD) and Becker (BMD) muscular dystrophy, we have studied the inheritance of loci, defined by DNA probes, linked to the disease locus in three generation families where the affected boy presented as an isolated case. Of 34 families analysed in this way, we have been able to determine that 15 boys have inherited their grandpaternal $X$ chromosome and 13 boys have inherited their grandmaternal $X$ chromosome. There are five boys who have inherited a recombinant grandparental $\mathrm{X}$ chromosome and in one case we have not been able to determine the origin of the mutant $\mathrm{X}$ chromosome. A recent theoretical analysis ( $\mathrm{Am} \mathrm{J} \mathrm{Hum}$ Genet 1986;38:827-40) has suggested that a very much larger sample size is needed before the results become statistically significant. Alternatively, a $100 \%$ accurate female carrier test would effectively reduce the number of families required.

Further linkage analysis of Emery-Dreifuss muscular dystrophy

C G COLE*, S V HODGSON*, A WALKER*, V DUBOWITZ $\dagger$, C GRANATA $\neq$, L MERLINI

*Paediatric Research Unit, Guy's Hospital, London SE1 9RT; +Department of Paediatrics and Neonatal Medicine, Hammersmith Hospital, London W12 OHS; and $\ddagger$ Istituto Ortopedico Rizzoli, Bologna, Italy.

It has previously been suggested by us (Hodgson et al, Hum Genet, in press) and others (J Med Genet, December 1986) that the locus for EDMD lies between Xq27 $\rightarrow$ Xqter. In order to strengthen existing data we screened three previously studied kindreds with a further seven polymorphic DNA probes recognising loci between $\mathrm{Xq} 24 \rightarrow$ Xqter. Overall a total of 18 DNA probes recognising 16 loci has now been tested on these families. The new data corroborate existing evidence for an Xq27 $\rightarrow$ Xqter localisation. In addition, we raise the possibility that
EDMD is the human homologue of the mouse muscular dystrophy gene $m d x$.

Sulphasalazine causes chromosome changes in patients with inflammatory bowel disease

J MCKAY, D P FOX, J BROWN, G HAWKSWORTH, AND

P W BRUNT

Departments of Genetics, Clinical Pharmacology, and Medicine, University of Aberdeen and Aberdeen Teaching Hospitals.

Sulphasalazine remains the treatment of choice for the long term management of colonic inflammatory bowel disease (IBD). We have shown that patients treated with sulphasalazine have a higher frequency of sister chromatid exchange (SCE) and micronucleus formation in their $\mathrm{N}$ lymphocytes than age and sex matched controls. This or indicates a raised frequency of DNA damage and chromo- 을 some aberrations or non-disjunction. In order to distinguish between the effect of the disease and of the drug in the causation of this effect, we have examined 25 IBD patients on diagnosis but before treatment and at intervals over a 21 month period during sulphasalazine or other therapy. There is a progressive increase in both SCE and micronucleus frequency in patients receiving sulphasalazine but no increase in the other patients. This implies that sulphasalazine is the cause of the increased level of genetic damage. Rate of acetylation of sulphapyridine, a major metabolite of sulphasalazine, is under genetic control but 'fast' and 'slow' acetylating subjects do not differ in their (raised) frequency of genetic damage. The significance of this genetic damage is as yet unclear but could be related to cancer risk. Alternative therapies for IBD should now therefore be similarly assessed in order to minimise risk to patients.

Urgent use of DNA typing to avoid prenatal diagnosis in a family at risk of cystic fibrosis

J BURN, D BROCK, A CURTIS, S HOLLOWAY, AND R NELSON Departments of Human Genetics, University of Newcastle upon Tyne and University of Edinburgh.

Two brothers married two sisters. When one couple produced a child with cystic fibrosis the second couple $\bar{N}$ were referred for counselling. The 1 in 16 recurrence risk $G$ was explained together with the difficulties presented by $\mathrm{N}$ second trimester prenatal diagnosis, a substantial false positive rate in cases of intermediate or low prior $\sigma$ probability. While the possibility of the use of linked DNA markers was being explored the couple presented with an $\stackrel{0}{ᄃ}$ unplanned pregnancy. Before the end of the first trimester $\mathbb{D}$ it was possible to demonstrate, using the probes $\mathrm{pJ} 3 \cdot 11$ and met $\mathrm{H}$ together with the restriction endonuclease $M s p \mathrm{I}, \underset{7}{T}$ that the sister had not inherited the same chromosome 7 as her affected niece. At that time, Spring 1986, a crossover $\stackrel{\mathbb{Q}}{\mathscr{Q}}$ rate of $3 \%$ was quoted which represented a risk of 1 in 94 D্ using the formula $3 \theta(1-\theta)^{2}+\theta^{3}$. The pregnancy has continued without intervention. The crossover rate of $1.5 \%$ 
now quoted reduces the risk of an affected child to 1 in 183 . This unusual family illustrates the value of exclusion tests in families at intermediate risk of cystic fibrosis.

\section{Chorionic villus sampling (CVS) in Fanconi anaemia} M MURER-ORLANDO, J LLERENA JR, L ZAHED, M MCGUIRE, M BOBROW, E C GORDON-SMITH*, AND C M RODECK $\dagger$ Paediatric Research Unit, Guy's Hospital, London SEI 9RT; *Royal Postgraduate Medical School, Hammersmith Hospital, London W12 OHS; and IInstitute of Obstetrics and Gynaecology, Queen Charlotte's Maternity Hospital, London W6 OXG.

Chromosome instability is one of the features which characterises Fanconi anaemia (FA). Chromosome breaks have been observed in both lymphocytes and fibroblasts of affected patients. Moreover, detection of FA fetuses has been reported on analysis of amniotic fluid and CVS (Auerbach, 1985, 1986). In our laboratory, a study has been conducted to establish the normal level of spontaneous and diepoxybutane (DEB) and mitomycin C (MMC) induced breakage in chorionic villus samples. In addition, two CVS samples from patients at risk of FA (A: first trimester; B: second trimester) have been analysed on direct, semi-direct, and culture preparations, using normal CVS and FA fibroblast cultures as controls. Chromosome breakage rates of the two 'at risk' fetuses was within the normal range. For patient $\mathrm{B}$, this result has been confirmed by the analysis of amniotic fluid and fetal blood. In due course, fetal blood sampling will be performed on patient $A$ to verify the CVS result.

\section{X chromosome breakpoint near the DMD locus: molecular and cytogenetic evaluation}

S HOLT*, R LINDENBAUM $\dagger, \mathbf{N}$ BLACKWELL $\dagger$, E MUNRO*, I CRAIG*, AND Y BOYD*

*Genetics Laboratory, Department of Biochemistry, Oxford; and †Medical Genetics Unit, Churchill Hospital, Oxford.

A girl patient with odd facies and mild neurological abnormalities has been found to have a reciprocal translocation $t(X ; 8)(p 11 \cdot 4 ; q 24 \cdot 2)$. Because of its potential usefulness in delineating the lower boundary of the DMD region in Xp21, we have undertaken a detailed cytogenetic and molecular evaluation of this breakpoint. In situ hybridisation of L1.28 (DXS7), 754 (DXS84), B24 (DXS67), and D2 (DXS43) to replication banded metaphase chromosomes demonstrated that $D X S 7$ and $D X S 84$ lie on the translocated $X$ chromosome $(\mathrm{tX})$ and that $D X S 67$ and DXS43 lie on the translocated 8 (t8). Of particular interest was the mapping of $D X S 84$, as this sequence is absent in the DNA of 'BB', a male DMD patient with a cytologically detectable minor Xp21 deletion (Am J Hum Genet 1985;37:250). Results from probing DNA from somatic cell hybrids which retained either the tX (WLOS 53C1) or the t8 (LOSAG 4-3R) confirmed that
$D X S 7$ and $D X S 84$ lie on the $\mathrm{tX}$ chromosome together with $O T C$. A further five sequences (DXS142, JMD HIP25, pXJI, DXS164), also absent in the 'BB' deletion, were shown to lie on the 18 . No evidence was found for any deletion or rearrangement of $\mathrm{X}$ chromosome material. These results indicate that $D X S 84$ may map more proximally than previously suggested; this could explain the higher than expected recombination rate observed between $D X S 84$ and $D M D$.

PRUFILE: a microcomputer database for clinical genetics D E MUTTON, K CHOWN, A C BERRY, L THOMSON, H HASSALL, AND M BOBROW

South East Thames Regional Genetics Centre, Paediatric Research Unit, Guy's Hospital, London SE1 9RT.

PRUFILE is a multi-user relational database designed to increase the efficiency of the offices and laboratories of a Clinical Genetics Unit. The programme is designed to be operated by secretarial and clerical staff, and to absorb all the essential background information on patients referred to the Unit. As well as providing a rapid 'look-up' system, it allows the printing of various documents essential to the smooth running of the clinic and laboratory-labels, referral documents, laboratory proformae, etc. The paged format of the database allows the linkage of individual records within a family (and thus provides the basis for a genetics register) and the production of facts and figures for reports and research.

Application of a cDNA probe for von Willebrand factor in families with von Willebrand disease

C SHIACH, K SPOWART, J M CONNOR, A F PETTIGREW, AND I M HANN

Departments of Medical Genetics, Haematology, and Medicine, University of Glasgow, Glasgow.

Eleven families with von Willebrand disease were studied with a cDNA probe (pvWF1100) for von Willebrand factor. This probe detects a $B g l$ II RFLP with fragment sizes $9 \mathrm{~kb}$ and $7.4 \mathrm{~kb}$ in addition to two invariant bands of $13 \mathrm{~kb}$ and $4.9 \mathrm{~kb}$. Four subjects from three families showed replacement of one $13 \mathrm{~kb}$ band by a fragment of $15 \mathrm{~kb}$. This was heritable and, as two subjects were normal family members, this is likely to be a new polymorphism. Otherwise no evidence was found to support gross gene rearrangements or deletions in these families. In 10 families autosomal dominant inheritance was apparent and in five informative meioses no recombination was observed between the $v W F$ locus and the disease trait. In the other family the parents, who are Asian first cousins, are clinically normal but one of their three children had severe von Willebrand disease. Detailed testing showed haematological evidence of mild von Willebrand disease in both parents and one of the two other children. Both parents were heterozygous for the vWF probe. The severely affected child was homozygous for the $9 \mathrm{~kb}$ fragment, the mildly affected child was heterozygous, and the normal 
child was homozygous for the $7.4 \mathrm{~kb}$ fragment. These findings would support the conclusion that at least some families with apparent autosomal recessive von Willebrand disease represent homozygosity for a mild dominant trait.

Application of two intragenic and two extragenic probes for carrier detection and early prenatal diagnosis in haemophilia A

C SHIACH, J M CONNOR, L PIRRIT, A F PETTIGREW, I M HANN, AND C D FORBES

Departments of Medical Genetics, Haematology, and Medicine, University of Glasgow, Glasgow.

Sixteen families segregating for haemophilia A were studied with two intragenic factor VIII probes (exons $17-18, F 8 A$, and exon 26) and two tightly linked extragenic probes (DX13, DXS15 and St14, DXS52). Thirty-eight obligate carriers were tested and $55 \%$ were informative with one or both intragenic probes. Addition of the extragenic probes rendered $89 \%$ informative, but for St 14 there were four recombinations in 29 phase known meioses. For DX13 no recombinants were observed in 14 phase known meiosis. Seventy-one females at risk were tested and $23(32 \%)$ could be offered definitive genetic counselling with the intragenic probes. A further $28(40 \%)$ could be given carrier risks on the basis of information from extragenic probes. In 20 females DNA analysis was unhelpful either because of maternal non-information (9) or unavailability of one or both parental samples (11). During the course of this study one obligate carrier requested first trimester prenatal diagnosis. This has predicted a female carrier fetus by a combination of chromosomal analysis, and DNA analysis with $F 8 A$ and a Y specific probe (GMGY7).

Peripheral neurofibromatosis: progress in mapping the disease locus

S M HUSON, M UPADHYAYA, M SARFARAZI, J H EDWARDS, AND P S HARPER

Section of Medical Genetics, University of Wales College of Medicine, Cardiff; and Genetics Laboratory, University of Oxford.

The mapping of the gene for peripheral neurofibromatosis (NF) will be an important step towards our eventual understanding of disease pathogenesis and its relationship to other forms of neurofibromatosis. Since our earlier study (Huson et al, J Med Genet 1986), which excluded the possibility of close linkage to myotonic dystrophy, we have obtained more negative data for a number of RFLPs. We have combined negative published results (Spence et al, $J$ Med Genet 1983; Dunn et al, Am J Hum Genet 1985; Spence et al, Darby et al, Seizinger et al, Ann NY Acad Sci, in press) with our own to produce an exclusion map for NF. For 28 markers on 13 chromosomes, lod scores are significantly negative. A pictorial exclusion map is presented using the computer programme EXCLUDE. This calculates the likelihood distribution of a newly tested marker around a single locus against the various map distances and gives the relative chance that the marker is in this position. Although the NF gene has not yet been localised, the combined data presented has already excluded a significant proportion of the genome and illustrates the value of collaborative studies to maximise the information. Pooling of data will also allow the detection of genetic heterogeneity in NF.

Sibs with severe congenital microcephaly, retarded growth and multiple chromosome mosaicism

J L TOLMIE, E B BOYD, M E FERGUSON-SMITH, P BATSTONE, AND J M CONNOR

The Duncan Guthrie Institute of Medical Genetics, $\stackrel{\mathrm{N}}{ \pm}$ Yorkhill, Glasgow G3 8SJ.

The first affected child was a female infant born to healthy, unrelated parents. Intrauterine growth retardation and $\frac{\text { O }}{3}$ microcephaly were detected by ultrasound scanning at 35 weeks' gestation. The birth weight at 37 weeks was $2.34 \mathrm{~kg}$ D and the OFC was $27 \mathrm{~cm}$. Chromosome analysis on 을 peripheral lymphocytes revealed two cell lines: 13 cells were apparently normal $46, \mathrm{XX}$ while seven cells were $47, \mathrm{XX},+18$. Mosaic trisomy 18 was diagnosed and a low recurrence risk given; despite this, prenatal chromosome analysis was requested in their next pregnancy. Two attempts at CVS failed and three attempts at amniocentesis were required. Chromosome analysis was as follows: five cells from two primary in situ cultures gave 46,XY 응 karyotypes: 15 cells, all from subcultures, had gain or loss $\varrho$ of various chromosomes. At term an abnormal male $\vec{F}$ infant, birth weight $1.64 \mathrm{~kg}$, OFC $25.5 \mathrm{~cm}$, was born. Skin 윽 fibroblast chromosome analysis in primary in situ cultures confirmed a $46, \mathrm{XY}$ karyotype, but three separate chromosome analyses in blood gave a high frequency of aneuploid cells. Re-evaluating the sister, chromosome analysis of primary in situ cultures from skin biopsy revealed a mosaic karyotype; three cells out of 18 with trisomy 2 in two cultures and three cells out of 18 with trisomy 21 in one culture. Twelve cells out of 18 in the three cultures had a 8 normal 46,XX karyotype. Analysis of lymphocytes also gave a high incidence of aneuploid cells. A possible 0 explanation is these infants are homozygous for a mutation which causes malsegregation of human mitotic chromosomes.

Plot 2000: a universal pedigree plotting programme G WOLAK AND M SARAFARAZI Section of Medical Genetics, University of Wales College of Medicine, Cardiff.

The programme is written in dBASE III and will run on any IBM compatible computer. Data for the programme is extracted from dBASE III files (or registers) or ASCII text $\frac{P}{\mathbb{D}}$ files and are in the same format as those used by LIPED and the LINKAGE programmes. The user has the opportunity to specify the exact type of pedigree to be drawn, indicating such quantities as the number and colour 
of probes, the size of the pedigree, and colour of symbols. These options are sorted for further use in a standard specification file. Multiple marriages in any generation can be drawn. There is no limit to the number of spouses for a subject or to the number of multiple marriages within a pedigree. The programme has been designed to have an unlimited capacity; pedigrees of over 2000 people have been created. There is a fixed limit of nine generations per pedigree. When a pedigree is run, a workfile and an output file is produced. The workfile can be re-used for later variations of the same basic pedigree and the output file can be used to draw several copies of the same pedigree without having to re-run the programmes. The output file is an HPGL (Hewlett Packard Graphics Language), which is standard for most plotters. The combination of a specifications file, a label/probe file, and a batch control file makes the system very flexible and powerful with a large number of variations possible. The programme is under continuous test and development and can be easily modified to accommodate new symbol variations and types of pedigree plot.

The following abstracts presented at the previous meeting of the Society have been carried over from the earlier issue owing to lack of space.

Autosomal dominant syndrome of ectodermal dysplasia and clefting

M TOLAROVÁ

Institute of Experimental Medicine, Czechoslovak Academy of Sciences, Prague, Czechoslovakia.

Combination of ectodermal dysplasia and clefting is quite usual in the EEC syndrome (ectrodactyly-ectodermal dysplasia-clefting). We have observed a mother and her son affected with a similar syndrome but lacking ectrodactyly-cleft palate, dystrophic teeth, alopecial dystrophic hair, and anodontia/dystrophic nails. Apparently this syndrome has an autosomal dominant pattern of inheritance with variable expression of symptoms. As far as we know there have been only a few cases reported so far.

\section{Phenotypic features of a possible $8 p$ trisomy segregating in a family \\ D KUMAR, A ClARK, M A CRESSWELL, AND C E BLANK Department of Genetics, Sheffield.}

A family is reported in which five out of seven children each have a high prominent forehead, coarse facies, bulbous nose, thick fleshy lips, and growth failure. Of these, four show moderate to severe mental retardation. Cytogenetic investigation revealed an abnormality of the short arm of chromosome $8(8 p+)$ in all of them. The other two sibs have normal chromosome complements. This apparently unbalanced chromosomal rearrangement was inherited from the mother, who has a strikingly similar facial configuration and is known to be mentally retarded. Cytogenetic and clinical findings suggest partial duplication of the short arm of chromosome 8 (dup8p23·1 $\rightarrow$ 8pter).

\section{An unusual case of segmental neurofibromatosis}

O W J QUARRELL, S M HUSON, I MCQUEEN, AND P S HARPER Section of Medical Genetics, University of Wales College of Medicine, Cardiff.

A 60 year old lady presented with a 30 year history of bilateral weakness and wasting of L234 myotomes; she was anaesthetic in the L234 dermatomes and had neurofibromata confined to these areas. There was no family history of neurofibromatosis. Segmental neurofibromatosis has previously been reported, but the unusual features of this case are that it is bilateral and associated with neurological deficit. Can the findings in this lady be explained by a single somatic mutation?

Testing candidate genes in the Marfan syndrome M KESTON AND R DALGLEISH

MRC Clinical and Population Cytogenetics Unit, Edinburgh, and Department of Genetics, University of Leicester, Leicester.

The detection of a collagen gene RFLP which segregates with the Marfan syndrome may indicate which gene is defective. The candidate genes implicated are: (a) collagen type I (chromosome 7). In one patient an elongated pro $\alpha 2$ (I) collagen chain has been detected (Byers et al, 1981). (b) Collagen type III (chromosome 2). This is a principal component of the adventitia and media of the wall of the aorta. (c) Elastin gene. Elastin is a major matrix component of arteries. Abnormal levels of the amino acids desmosine and isodesmosine were found (Abraham et al, 1982). Using collagen probes $\mathrm{NJ}-3$ ' and a subcloned $1.2 \mathrm{~kb}$ EcoRI fragment of the pro $\alpha 2(\mathrm{I})$ and pIII 54 and $1 \mathrm{dF}$ $17 / 1 \cdot 6 \mathrm{E}$ for the $\alpha 1$ (III) chain, we have excluded the $\alpha 2$ (I) and $\alpha 1$ (III) collagen chains as the cause of the gene defect in our larger Marfan families. Studies using an elastin gene probe will follow. A family pedigree is shown which illustrates the great difficulty in interpreting probe results. However, as some of these individual members may be homozygous for the Marfan gene, this may prove a powerful means of excluding large regions of the human genome as the locus of the mutant gene.

A family with two balanced translocations, three unbalanced segregants, and a carrier with $X X X$ constitution M A HAMILL, S H ROBERTS, P S HARPER, AND K M LAURENCE University of Wales College of Medicine, Cardiff.

The proband was a male with multiple congenital abnormalities including heart and lung defects, abnormal facies, and short limbs, who died in the neonatal period. He was shown to have partial trisomy $2 p$ due to an adjacent 1 segregation of a maternal balanced reciprocal transloca- 
tion, $t(2 ; 15)(p 23 \cdot 3 ; q 26 \cdot 3)$. The maternal aunt of the proband, who subsequently gave birth to a similarly affected female, was also found to carry the balanced translocation $\mathrm{t}(2 ; 15)(\mathrm{p} 23 \cdot 3 ; \mathrm{q} 26 \cdot 3)$ and, in addition, a balanced Robertsonian translocation, $t(13 q ; 22 q)$. Further family studies showed that the reciprocal translocation had been inherited from the maternal grandfather, whereas the Robertsonian translocation had been inherited from the maternal grandmother, who also had three $\mathrm{X}$ chromosomes. Partial trisomy $2 p$ has since been diagnosed prenatally in one of two pregnancies investigated in the mother of the proband. The karyotype was confirmed in lymphocyte and fibroblast cultures initiated from the abortus.

\section{An intrachromosomal insertion of chromosome 13 in a family with psychosis and mental subnormality $S$ H ROBERTS, V A COWIE AND K RAMESHWARI SINGH University of Wales College of Medicine, Cardiff.}

A family is reported in which an apparently balanced intrachromosomal insertion, inv ins(13)(q21.3q32q31), was detected in four members, three of whom show psychiatric disorder including mental subnormality, personality defects, and frank psychosis. The presence of the inversion in a mentally normal member of the family, however, appeared to exclude a causal relationship. A review of previously reported intrachromosomal insertions shows that in all but one case they were ascertained through abnormal offspring with imbalances resulting from recombinant chromosomes. These were generated by crossing over in the segment interstitial to the inserted segment and its site of origin. In the present case, the risk of abnormal offspring in a normal carrier of the inversion appears to be low because the interstitial and inserted segments are relatively short and an apparently low frequency of chiasmata might be expected within them.

\section{Homozygous paracentric inversion 12 in a mentally retarded boy \\ S H ROBERTS, H A PRICE, AND K M LAURENCE \\ University of Wales College of Medicine, Cardiff.}

A 17 year old boy was referred for cytogenetic investigation because of mental retardation. On later examination he was also found to be short and microcephalic with an unusual facies. He was shown to have a homozygous paracentric inversion, inv $(12)(q 15 q 23 \cdot 2)$, while his mother and father, who are first cousins, and his brother are heterozygous for the inversion. There are no previous reports of homozygotes for a paracentric inversion, and very few of homozygotes for any other structural rearrangement. The abnormal phenotype of the proband might be the result of homozygous gene damage or position effect, the involvement of other homozygous recessive alleles, or a very small duplication or deletion due to unequal crossing over. A review of published reports shows that carriers of putative paracentric inversions may be at risk of producing abnormal offspring with recombinant chromosomes, although in most cases these appear

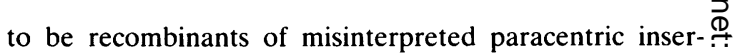
tions. There may also be an interchromosomal effect that $\vec{\nexists}$ increases the likelihood of aneuploidy in offspring of $\stackrel{\oplus}{?}$ carriers. Prenatal cytogenetic monitoring of paracentric inversion carriers is therefore advisable.

Prenatal detection of a male pseudohermaphrodite resulting from an $X ; Y$ translocation

$S$ H ROBERTS, D P DUCKETT, E LITTLE, AND K M LAURENCE $\vec{\circ}$ University of Wales College of Medicine, Cardiff.

An unbalanced $\mathrm{X} ; \mathrm{Y}$ translocation, $\mathrm{t}(\mathrm{X} ; \mathrm{Y})(\mathrm{p} 11 \cdot 2 ; \mathrm{p} 11 \cdot 2), \stackrel{\omega}{\mathrm{S}}$ was detected in the fetus of a woman referred for $\overline{\bar{B}}$ amniocentesis because of a high serum AFP level. Since there was one normal $X$ chromosome, the fetus had a duplication of Xp11.2 $\rightarrow$ Xpter and deletion of $A$ Yp11.2 $\rightarrow$ Ypter. The prenatal karyotypes were normal. On is termination of the pregnancy, the fetus appeared to be a $r$ relatively normal female with clitoromegaly. Internally, 음 the vagina and partially bicornuate uterus were hypo-plastic, and although the fallopian tubes were normal, the $\rightarrow$ gonads were testes with associated epididymides. The $\mathrm{X} ; \mathrm{Y}$ 음 translocation was also found in fetal lymphocyte and lymphoblast cultures. The presence of testes supports the $\overrightarrow{0}$ view that a gene directing testicular development is located in the proximal, rather than the distal, half of $\mathrm{Yp}$. However, the predominantly female phenotype is indica-o tive of defective genetic control of sexual differentiation. This may be due to a failure to achieve dosage compensa- $\bar{O}$ tion for Xp gene, but, in addition, the testis determining gene on Yp may have been adversely affected by the $\stackrel{\mathbb{Q}}{\varrho}$ rearrangement, or gene deletion may have occurred if more than one $\mathrm{Yp}$ gene is involved in testicular function.

A mentally retarded male with a 49,XXXYY karyotype R F WHEATER, $V$ A COWIE, AND $K$ R SINGH University of Wales College of Medicine, Cardiff.

During the course of a cytogenetic survey of a hospital for the mentally retarded, a 42 year old male was found to have the karyotype $49, \mathrm{XXXYY}$, which has been reported 3 on only three previous occasions. The clinical features of the proband had much in common with these cases. He 3 had a eunuchoid habitus with moderate gynaecomastia and $D$ a female distribution of body fat and hair. He possessed an overhanging frontal bone, deep set eyes, and a large $\mathrm{N}$ prognathic lower jaw. There are at least four sequences of ${ }^{\mathrm{r}}$ maternal and paternal meiotic non-disjunction events $N$ which would result in a 49, XXXYY chromosome comple-N ment. In addition, premeiotic non-disjunction, post- $-\sigma$ fertilisation non-disjunction, and single chromosome re plication are non-meiotic events which could be involved info the formation of this aberrant karyotype.

A new variant of chromosome 16 P W THOMPSON AND S H ROBERTS University of Wales College of Medicine, Cardiff.

We report four cases of a probable new variant of 
chromosome 16, which show additional material in the proximal region of the short arm. The $16 p+$ chromosome appears metacentric, the extra material showing three bands with GTG and Q banding, and negative staining with $\mathrm{C}$ banding, Distamycin/DAPI, and silver staining. Two of the probands with $16 \mathrm{p}+$ were diagnosed prenatally and in both instances the variant was present in a normal parent. The outcome of one of these pregnancies was a normal male infant while the other pregnancy is continuing. One index case was a three year old male referred with developmental delay and dysmorphic features. Investigation of his family showed the $16 p+$ was present in the father, grandmother, and four other phenotypically normal relatives. The fourth index case was a 25 year old female presenting with primary amenorrhoea who did not consent to family studies. These findings suggest that the $16 p+$ is a variant and is unlikely to be of clinical significance. The $16 \mathrm{p}+$ appears to be similar to the reported variants of chromosome 9 that have additional $\mathrm{C}$ band negative material in the proximal region of $9 p$. 